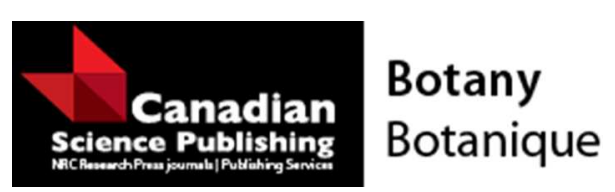

\title{
Comparative development of simple and compound leaves in the genus Cecropia
}

\begin{tabular}{|r|l|}
\hline Journal: & Botany \\
\hline Manuscript ID & cjb-2016-0148.R1 \\
\hline Manuscript Type: & Article \\
\hline Date Submitted by the Author: & 26-Aug-2016 \\
\hline Complete List of Authors: & $\begin{array}{l}\text { Ogden, Michael; University of Prince Edward Island, Biology } \\
\text { Lacroix, Christian; University of Prince Edward Island, Biology }\end{array}$ \\
\hline Keyword: & $\begin{array}{l}\text { leaf development, compound leaf, simple leaf, primordia, divergence angle } \\
\text { and plastochron ratio }\end{array}$ \\
\hline \multicolumn{2}{|l}{} \\
\hline
\end{tabular}

SCHOLARONE ${ }^{\text {IM }}$

Manuscripts 
Comparative development of simple and compound leaves in the genus Cecropia

Michael S. Ogden and ${ }^{1}$ Christian R. Lacroix Department of Biology, University of Prince Edward Island, 550 University Avenue, Charlottetown, PE, Canada C1A 4P3

${ }^{1}$ Corresponding author: lacroix@upei.ca 


\begin{abstract}
Plants develop leaves that range from simple to compound in shape. The evolutionary divergence of simple and compound leaves has spurred research into identifying cellular and molecular processes involved in leaf shape determination. The roles of various genes and signalling pathways have been characterized in specifying leaf shape, however, few studies have investigated leaf primordium structure and shoot apex organization throughout development of both simple and compound leaves. Using Cecropia obtusa and Cecropia sciadophylla, two putatively closely related species bearing simple palmate and palmately-compound leaves, respectively, we compared the morphogenesis of leaves of both species at the shoot apex. Analysis of shoot apices using scanning electron microscopy yielded a non-significant difference in leaf primordium divergence angles and plastochron ratios, suggesting that divergence of the two leaf types occurred independently of primordium organization and growth rate at the shoot apex. Qualitative analysis of primordium initiation and morphogenesis revealed that both species share highly homologous development, as primordium structure and lobe/leaflet initiation sites are complementary in both leaf types. Our observations suggest a high degree of conserved ontogeny in the developmental pathways underlying the morphogenesis of simple palmate and palmately-compound leaves in these two species.
\end{abstract}

Key words: leaf development, Cecropia sciadophylla, Cecropia obtusa, compound leaf, simple leaf, primordia, divergence angle, plastochron ratio 


\section{Introduction}

Angiosperms display an abundant diversity of leaves of different shapes and sizes. Phenotypes vary widely between species based on a vast range of characteristics. For example, margin serration, lobation, fenestration, and venation are just a few of the many variable parameters present in mature leaf forms. Despite the wide range of morphological differences in leaves across species, every leaf can be classified into one of two categories: simple or compound. A simple leaf consists of one entire unit with a single lamina, whereas a compound leaf consists of multiple joined units (leaflets) (Harevan et al. 1996). To date, it remains unclear whether the ancestral angiosperm bore simple or compound leaves, although a growing body of evidence suggests that the first leaves were simple in structure, with some species having the capability of converting to compound leaves and reverting to simple leaves throughout their evolution (Floyd and Bowman 2010; Geeta et al. 2012). This longstanding question has spurred an interest in understanding the divergence of simple and compound leaf morphologies from both an evolutionary and developmental perspective.

For decades, researchers have been investigating the similarities and differences between simple and compound leaf development. A general theory proposed by Arber (1950) suggests that compound leaves share some degree of developmental homology to shoots (partial-shoot theory). In recent years, advances in molecular and genetic analysis have led to increasing evidence that supports the partial-shoot theory through investigation of the shoot apical meristem (SAM) in a variety of eudicots (Sinha 1999; Bharathan et al. 2002; Champagne and Sinha 2004; Bar and Ori 2014). 
The SAM is a region of indeterminately dividing, undifferentiated cells found at the apex of plant shoots, and gives rise to all aerial plant tissue, including leaves. Although many factors are likely involved in leaf initiation, development, and morphogenesis (Bar and Ori 2014), studies have revealed that cellular signaling pathways (Dodsworth 2009), fluctuating hormone levels (Reinhardt et al. 2003; Fambrini and Pugliesi 2013), and specific patterns of gene expression (Champagne and Sinha 2004; Rast-Somssich et al. 2015) are key components in these developmental processes. For example, Class I KNOTTED1-LIKE HOMEOBOX (KNOX1) is continuously expressed in the SAM and acts locally to regulate development and maintenance of structure and function (Uchida et al. 2010). During leaf primordium initiation at the flanking region of the SAM, KNOX1 expression is normally absent and remains unexpressed throughout development, morphogenesis, and maturation of simple leaves. Conversely, in compound leaf development across most eudicots, KNOX1 expression is upregulated in the leaf primordium following initiation, leading to leaflet development, and expression eventually ceases, leading to leaf maturation (Bharathan et al. 2002). Overexpression of KNOX1 in alfalfa plants results in increased leaflet production (Champagne et al. 2007), and KNOX1 downregulation in tomato plants leads to decreased leaflet production (Kim et al. 2003). Additionally, high concentrations of auxin in localized areas of the SAM downregulates the expression of KNOX1, and is thought to play a key role in leaf primordium initiation (Shani et al. 2006). KNOX1 is not the only factor responsible for the development of leaf shape. In fact, recent studies by Tsiantis and colleagues (Rast-Somssich et al. 2015; Vlad et al. 2014) and a comprehensive review by Bar and Ori (2016) reveal a complex genetic network 
where "different developmental stages within a given program are often controlled by overlapping sets of factors or 'tools', thus comprising the 'toolbox' of leaf development".

Even though many studies to date have investigated the role of genes and hormones in leaf shape development and morphology in individual species, there is a paucity of scientific literature surrounding comparative developmental studies of closely related species bearing both simple and compound leaves. The study of closely related species offers the ability to compare phenotypes between relatively homologous genomes, enabling the parallel investigation of gene expression, alleles, and mutations, which can identify the underlying genetic factors responsible for each phenotype (Frankel et al. 2011; Hay et al. 2014). There are a few well-documented cases of closely related species bearing variable leaf morphology, from simple to compound leaves. For example, Ampelopsis cordata and Ampelopsis arborea bear simple and compound leaves, respectively (Jones et al. 2013), as do Arabidopsis thaliana and Cardamine hirsuta (Barkoulas et al. 2008; Rast-Somssich et al. 2015). Our study highlights the importance of investigating the ontogenetic processes involved in the initiation of divergent leaf forms to make better developmental and genetic predictions of how leaf forms diverge. We want to explore to what extent similar morphogenetic developmental pathways can lead to markedly different leaf forms using two closely related, yet phenotypically distinct species, Cecropia obtusa Trécul and Cecropia sciadophylla Martius.

Cecropia Loefl. is a genus of pioneer tree species native to and distributed across the Neotropical ecozone (Berg and Franco Rosselli 2005). There are currently 61 identified species of Cecropia, although it is predicted that upwards of 80 species 
exist (Berg and Franco Rosselli 2005; Wu et al. 2013). Cecropia obtusa and Cecropia sciadophylla prove to be interesting species as they are found within the same genus but have contrasting leaf morphologies. Cecropia obtusa develops a palmately lobed leaf (Heuret et al., 2002; Berg and Franco Rosselli 2005) whereas C. sciadophylla develops a palmately compound leaf (Berg and Franco Rosselli 2005; Letort et al. 2009) (Fig. 1). A number of phylogenetic analyses have placed Cecropia within the family Urticaceae, although data on the phylogenetic distance between individuals within Cecropia is limited (Savolainen et al. 2000; Sytsma et al. 2002; Wu et al. 2013; Treiber et al. 2016). Wu et al. (2013) proposed that the members of Cecropia have the potential to form a natural group, a theory which has long been presumed by taxonomists (Bonsen and Ter Welle 1983). Although the phylogenetic relationship between Cecropia obtusa and Cecropia sciadophylla has not been analyzed to date, their distinct morphological characteristics clearly place them within Cecropia (Bonsen and Ter Welle 1983). However, a recent study by Treiber et al. (2016) reports that $C$. sciadophylla is part of an early diverging lineage that is closely related to Musanga.

The aims of this study are: (1) to compare early stages of development of $C$. obtusa and C. sciadophylla using qualitative and quantitative data at the microscopic scale, and (2) to characterize the developmental progression of each species beginning with leaf initiation, to highlight the extent to which similar morphogenetic stages can lead to the development of divergent leaf forms.

\section{Materials and methods}




\section{Plant material and tissue fixation}

In 2012, shoot apices of Cecropia obtusa and Cecropia sciadophylla were collected from French Guiana, South America. Individual plants were identified to the species level, and shoot apices were harvested from the plants. They were immediately fixed in a solution of FAA (formalin - glacial acetic acid - absolute ethanol, 1:1:9 (by volume)), as outlined in Jeune and Lacroix (2009), in order to preserve the samples. Samples were then transported to the University of Prince Edward Island where they were stored at room temperature in FAA.

\section{Scanning electron microscopy}

To prepare the fixed shoot apices for scanning electron microscopy, nine apices belonging to $C$. obtusa and 11 belonging to $C$. sciadophylla were removed from the fixative solution and were soaked in a solution $70 \%$ ethanol with acid fuchsin to stain the tissues overnight. This type of staining improves dissection accuracy and precision near the SAM by increasing the visibility of primordia and associated structures. The samples were then placed in a petri dish filled with $70 \%$ ethanol and dissected under an Olympus SZ40 stereomicroscope (Olympus Canada Inc., Richmond Hill, Ontario, Canada) to reveal the SAM and up to the first four youngest leaf primordia. Following dissection, the samples were placed in $90 \%$ ethanol for a minimum of 12 hours. The samples were subsequently transferred to absolute ethanol for two hours. They were then processed in a model 2800 LADD critical point dryer (LADD Research, Williston, Vermont, USA) which uses $\mathrm{CO}_{2}$ as a transitional fluid to completely dehydrate the samples without disrupting their cellular structure. The critical point dried samples were then mounted on 
aluminum SEM specimen mounts using adhesive tabs with silver paint being used to ground them. A Denton Vacuum Desk II sputter-coater (Denton Vacuum, Moorestown, New Jersey, USA) was used to coat the samples with $300 \AA$ of gold-palladium. A number of critical point dried samples were further dissected to reveal sequentially earlier stages of development using a fine dissection needle. A Hitachi TM3000 Tabletop SEM (Hitachi High-Technologies, Toronto, Ontario, Canada) was used to view the samples as they were further dissected and to capture images.

\section{Phyllotactic measurements}

Standard phyllotactic parameters were used to quantitatively compare the development of leaves of both species. Divergence angles and plastochron ratios were calculated for the shoot apices of nine and 11 individuals of $C$. obtusa and $C$. sciadophylla, respectively. The divergence angle is the angle between two successive leaf primordia using the center of the SAM as a point of reference, and the plastochron ratio is the ratio of the distance from the center of the SAM to the centers of two successive leaf primordia. These parameters were calculated with the use of 3D-Image Viewer (Version 2.1.0, Denshi Kougaku Kenkyusyo Co., Ltd.), a SEM image analysis program. All data were analyzed and compared using two-sample t-tests.

\section{Results}




\section{Initiation and morphogenesis of leaf primordia}

In both Cecropia obtusa and Cecropia sciadophylla, leaf primordia are initiated singly along the flanks of the shoot apical meristem (SAM), initially appearing as a small rounded bump (Figs. 2A and 2B). Across shoot apices of both species, the site of primordium initiation is located at a mean distance of $118.50 \pm 23.29 \mu \mathrm{m}$ from the center of the SAM; there is no significant difference between the mean value for each species $(n=20)$. Following initiation, the leaf primordium of each species assumes a similar conical, somewhat pyramidal shape (Figs. 2C and 2D), and stipule initiation begins at the base of the adaxial surface of the primordium prior to lobe and leaflet initiation (Figs. $2 \mathrm{E}$ and $2 \mathrm{~F})$. The shape of each species' leaf primordium remains similar until the adaxial surface reaches approximately $200 \mu \mathrm{m}$ from base to tip (coinciding with the P2 stage of development) where, at this point, lobe and leaflet initiation begins (Figs. 2E to $2 \mathrm{H}$ ). For the purposes of referring to developmental stages, the youngest primordium will be labeled as $\mathrm{P} 1$, followed by the second youngest primordium $\mathrm{P} 2$ and so on.

\section{Lobe and leaflet initiation and development}

Each species first initiates three lobes or leaflets in the P2 stage of development, where two units develop on opposite sides of a central unit. In C. obtusa, the two lobes (first pair) that are initiated on either side of a more prominent central lobe arise in the midsection of the primordium, whereas in C. sciadophylla the first pair of leaflets is initiated at approximately the same level as the central leaflet (Figs. 2E and 2F). Up until this stage of development, the leaf primordia of both species are still relatively similar in morphology. All consecutive lobes/leaflets are initiated in pairs, with each unit being 
inserted on opposite sides of the primordium, directly below the preceding unit. Following the initial three lobe/leaflet state, leaflet initiation occurs at a faster rate than lobe initiation but lobe margins begin to curl inward towards the adaxial surface before leaflets do (Figs. $2 \mathrm{G}$ and $2 \mathrm{H}$ ). Leaflet margins begin to curl in a similar fashion to lobe margins following the initiation of the third pair of leaflets. The end of the morphogenetic phase is marked by the completion of a full circular pattern of lobes and leaflets on the adaxial surface of each leaf type (Figs. $2 \mathrm{I}$ and $2 \mathrm{~J}$ ), which consistently occurred in the P4 stage of development in both species. At the end of morphogenesis, C. obtusa displayed from six to eight lobes, and C. sciadophylla, displayed from 12 to 15 leaflets. These numbers are consistent with the number of lobes and leaflets reported when leaves reach maturity, with $C$. obtusa displaying from six to nine lobes, and $C$. sciadophylla from seven to 15 leaflets (Berg and Franco Rosselli 2005). Although each species develops lobes and leaflets of different numbers and size at the end of morphogenesis, they both display the same basic folded morphology.

The overall organization of leaf primordia at the shoot apex shows a high degree of similarity between each species. They both develop teardrop-shaped stipules of comparable size and structure, differing mainly in a more pronounced, "sharp" margin in C. sciadophylla, compared to the rounded margin of C. obtusa (Figs. 3A to 3D). Individual leaves arise singly at the edge of the SAM in a spiral fashion (Figs. $3 E$ to $3 \mathrm{H}$ ). Clockwise spirals (Fig. 3F) and counterclockwise spirals (Fig. 3E) were observed to occur in both species. When apices are fully dissected, the arrangement at the shoot apex appears to be superimposable between each species. The four primordial developmental stages (P1-P4) are aligned at the same position on the apex of each 
species (Fig. 3), which is further verified through comparing divergence angles and plastochron ratios. Additionally, trichome initiation occurs prior to the end of the morphogenetic phase of leaf development, although trichome elongation in $C$. sciadophylla proceeds at a faster pace than in C. obtusa, with trichomes in C. sciadophylla appearing to be longer and more abundant at the end of morphogenesis (Figs. $3 \mathrm{G}$ and $3 \mathrm{H}$ ). This variance in trichome development may be due to the nature of the type of trichome present in each species, as there is a diversity of trichomes in the Cecropia genus in which six distinct types have been characterized (Berg and Franco Rosselli 2005).

\section{Divergence angle}

Both C. obtusa and C. sciadophylla display a fluctuation in divergence angles between successive primordia in individual apices, from $141.26^{\circ}$ to $168.43^{\circ}$, and $137.78^{\circ}$ to $170.51^{\circ}$, respectively (Fig. $4 \mathrm{~A}$ ). The average divergence angle per apex ranged from $151.00^{\circ}$ to $155.78^{\circ}$ (C. obtusa), and $146.83^{\circ}$ to $162.34^{\circ}$ (C. sciadophylla). Individual apices of $C$. sciadophylla displayed a larger range of fluctuation in their average divergence angles $\left( \pm 15.51^{\circ}\right)$ compared to $C$. obtusa apices $\left( \pm 4.78^{\circ}\right)$. When divergence angles between successive leaf primordia are compared between species, only the mean divergence angle between the first and second primordium is significantly different (two-sample t-test, $p=0.03$ ). The difference in divergence angles between the 
primordia of each species is lower and less variable for older leaf primordia. Overall, the mean divergence angle for each species is not significantly different (two-sample t-test, $\mathrm{p}=0.52$ ), calculated as $153.48^{\circ} \pm 7.50^{\circ}$ and $152.07^{\circ} \pm 8.25^{\circ}$ for $C$. obtusa and $C$. sciadophylla, respectively.

\section{Plastochron ratio}

The plastochron ratio between successive leaf primordia in relation to each species of Cecropia fluctuated from 1.39 to 2.63 (C. obtusa), and 1.36 to 2.62 (C. sciadophylla) (Fig. 4B). The mean plastochron ratio per apex ranged from 1.80 to 2.04 (C. obtusa), and 1.62 to 2.18 (C. sciadophylla). Across individual apices of each species, C. sciadophylla displayed a wider range of fluctuation in the mean plastochron ratio per apex $( \pm 0.56)$ compared to that of $C$. obtusa $( \pm 0.24)$. No significant difference was found between the mean plastochron ratios of consecutive primordia when both species were compared. The mean plastochron ratio was not significantly different between species, measured as $1.90 \pm 0.31$ and $1.94 \pm 0.40$ (two-sample t-test, $p=0.65$ ) for C. obtusa and C. sciadophylla, respectively. 


\section{Discussion}

In the search to understand the evolution and molecular processes involved in simple and compound leaf development, a crucial yet often overlooked parameter of development is the morphogenetic phase of growth, which covers the period of time during which the initiation of lobes/leaflet takes place. This short-spanned phase occurs at the microscopic level and only accounts for a small percentage of the leaf's total developmental timeframe, yet it is central in establishing the overall morphology of the leaf (Jeune and Lacroix 2009). This further suggests that the timing of the morphogenetic versus the differentiation phases affect leaf development, an idea that is supported by mutant analysis, and seems to involve a complex interplay between KNOX, KNOX regulators such as TCP, and APETALA/FRUITFUL genes (Bar and Ori, 2014). Our data suggest no difference in the morphogenetic phase we covered between the two species, implying that the timing between morphogenesis and differentiation is unchanged in these simple versus compound leaf types. More subtle or specific developmental differences likely arise during the subsequent secondary morphogenetic and differentiation phases (sensu Floyd and Bowman 2010).

On the macroscopic scale, there are distinct morphological differences between the simple-leaved C. obtusa (even though it is lobed), and the compound-leaved $C$. sciadophylla (Fig. 1), yet a surprising degree of developmental homology is observed during leaf initiation and morphogenetic phase of development. In both species, leaves initiate singly at the edge of the SAM (P1) (Figs. 2A to 2D), and lobes/leaflets initiate in pairs sequentially down the margin of the primordium beginning during P2 (Figs. 2E to 2J). A period of additional lobe/leaflet initiation occurs during P3 (Figs. $2 \mathrm{G}$ and $2 \mathrm{H}$ ), and 
P4 marks the end of the morphogenetic phase when leaves complete a full circular complement of lobes/leaflets along the primordium margin (Figs. $2 \mathrm{l}$ and $2 \mathrm{~J}$ ). The transition from P1 to P4 occurs at the same rate and position in a spiral phyllotaxy (Figs. $3 \mathrm{E}$ and $3 \mathrm{~F}$ ) at the apex of each species. Additionally, lobes and leaflets both assume an inward-folding morphology, which is a characteristic typically seen in leaflets at the microscale. Together, these observations suggest a high degree of conserved ontogeny in the developmental pathways underlying leaf development. We further support the precision of these observations based on our phyllotactic analyses.

Plastochron ratios and divergence angles are well-established phyllotactic parameters used to compare leaf organization patterns and growth rates (Adler et al. 1997; Rutishauser 1998). In order to quantify our observations, we used these measurements and found striking similarity between both species. There is no significant difference in mean divergence angle between the species, although there is a significant difference when the P1-P2 divergence angles are compared, which is likely due to the considerable flux that takes place at the level of the SAM during leaf initiation (Fig. 4A). The divergence angles between P2-P3 and P3-P4 show stabilization with increased primordium age with non-significant differences in values between species. Additionally, there is no significant difference in plastochron ratios between primordia (Fig. 4A), indicating that both species initiate leaves at the same distance from the SAM and at the same rate. Together, these two phyllotactic parameters show that despite the difference in leaf forms at maturity, the simple and compound leaves studied share highly homologous development during initiation and throughout the morphogenetic phase of growth. 
When both qualitative and quantitative data are combined, our findings suggest that C. obtusa and C. sciadophylla (and possibly other members of Cecropia) have the potential to act as a valuable model system for studying the role of putative genetic pathways involved in the determination and extent of leaf dissection. This proposed Cecropia system provides advantages when compared to other model systems that require detailed knowledge of shoot apex structures and fine manipulation of complex, clustered apices. By simply removing subsequent stipules to the SAM level, a clear view of the SAM and surrounding primordia can be obtained with relative ease. As KNOX, $R C O$, and CUC genes appear to be heavily involved in determining complexity of leaf margins, the identification and manipulation of homologs of these genes in these species has the potential to show how gene upregulation and downregulation impacts respective leaf morphogenesis. Since leaf morphogenesis was similar in both of our species, investigating other mechanisms involved in leaf development post primary morphogenesis would also be of interest, especially as it pertains to distinguishing between the elaboration of a lobe, which is an integral part of the single leaf blade versus a leaflet, which is a distinct 'unit'. Based on the current state of knowledge, there is likely a continuum in the extent of local restriction or promotion of growth operating at the margin of leaves explaining the broad array of leaf forms within and between species (Bar and Ori 2014; Sicard et al. 2014; Vlad et al. 2014; Rast-Somssich 2015).

Our results highlight the high degree of morphological homology across multiple qualitative and quantitative variables which occurs during their initiation and morphogenetic phase of leaf development. These data infer that the evolutionary divergence between simple and compound leaf forms and the shoot apical meristem for 
that matter occurred while retaining homologous genetic elements involved in development (Floyd and Bowman 2010). This observation is also supported by a similar comparative study by Jones et al. (2013), who found that the simple-leaved Ampelopsis cordata and compound-leaved Ampelopsis arborea (Vitaceae) share highly homologous morphology during leaf initiation and early morphogenesis. Similar to our findings, Jones et al. (2013) highlighted key landmarks in morphogenesis of both leaf types which marked leaf form divergence, where each primordium maintained homologous shape and structure until the initiation of the first marginal serration in the simple-leaved species and the first lobe in the compound-leaved species, which marked morphogenetic divergence between the species.

The high degree of homology during the morphogenetic phase of growth observed in both our study and to a similar extent that of Jones et al. (2013) and Tsiantis and colleagues (Vlad et al. 2014; Rast-Somssich et al. 2015) may suggest that relatively noncomplex modifications to the developmental pathway of the leaf may be capable of converting a lobe to a leaflet, or vice-versa. This does, to a certain degree, support the partial shoot theory, which theorizes that the compound leaf is homologous in structure and development to a growing shoot, with plants being capable of switching between simple and compound leaf growth with "relative ease" because of similarities that exist in "developmental genetic mechanisms" involved in the growth and development of SAMs and leaves in eudicots (Floyd and Bowman 2010). By identifying key landmarks of homology and divergence during morphogenesis in related species, researchers can target specific developmental stages to further elucidate the ontogenetic pathways which give rise to different types of leaves. 


\section{References}

Arber, A. 1950. The natural philosophy of plant form. Cambridge University Press, Cambridge.

Adler, I., Barabé, D., and Jean, R. 1997. A history of the study of phyllotaxis. Ann. Bot. 80(3): 231-244. doi:10.1003/anbo.1997.0422.

Bar, M., and Ori, N. 2014. Leaf development and morphogenesis. Development. 141(22): 4219-4230. doi:10.1241/dev.106195. PMID:25371359.

Barkoulas, M., Hay, A., Kougioumoutzi, E., and Tsiantis, M. 2008. A developmental framework for dissected leaf formation in the Arabidopsis relative Cardamine hirsuta. Nat. Genet. 40(9): 1136-1141. doi:10.1038/ng.189. PMID:19165928.

Berg, C., and Franco Rosselli, P. 2005. Flora neotropica monograph 94: Cecropia. New York Botanical Garden Press, New York.

Bharathan, G., Goliber, T.E., Moore, C., Kessler, S., Pham, T., and Sinha, N. 2002. Homologies in leaf form inferred from KNOX1 gene expression during development. Science. 296(5574): 1858-1860. doi:10.1126/science.1070343. PMID:12052958. 
Bonsen, K., and Ter Welle, B.J.H. 1983. Comparative wood and leaf anatomy of the Cecropiaceae (Urticales). Bull. Mus. Natn. Hist. Nat. Section B, Adansonia. 5(2): 151177.

Champagne, C., and Sinha, N. 2004. Compound leaves: equal to the sum of their parts? Development. 131(18): 4401-4412. doi:10.1242/dev.01338. PMID:15342471.

Champagne, C., Goliber, T., Wojciechowski, M., Mei, R., Townsley, K., Paz, M., Geeta, R., and Sinha, N. 2007. Compound leaf development and evolution in the legumes. Plant Cell. 19(11): 3369-3378. doi:10.1105/tpc.107.052886. PMID:17993625.

Dodsworth, S. 2009. A diverse and intricate signalling network regulates stem cell fate in the shoot apical meristem. Dev. Biol. 336(1): 1-9. doi:10.1016/j.ydbio.2009.09.031. PMID:19782675.

Fambrini, M., and Pugliesi, C. 2013. Usual and unusual development of the dicot leaf: involvement of transcription factors and hormones. Plant Cell Rep. 32(6): 899-922. doi:10.1007/s00299-013-1426-1. PMID:23549933.

Frankel, N., Erezyilmaz, D., McGregor, A., Wang, S., Payre, F., and Stern, D. 2011. Morphological evolution cause by many subtle-effect substitutions in regulatory DNA. Nature. 474(7353): 598-603. doi:10.1038/nature10200. PMID:21720363. 
Floyd, S.K., and Bowman, J.L. 2010. Gene expression patterns in seed plant shoot meristems and leaves: homoplasy or homology? J. Plant Res. 123(1): 43-55. doi: 10.1007/s10265-009-0256-2. PMID:19784716.

Geeta, R., Dávalos, L., Levy, A., Bohs, L., Lavin, M., Mummenhoff, K., Sinha, N., and Wojciechowski, M. 2012. Keeping it simple: flowering plants tent to retain, and revert to, simple leaves. New Phytol. 193(2): 481-493. doi:10.1111/j.1469-8137.2011.03951.x. PMID:22091556.

Harevan, D., Gutfinger, T., Parnis, A., Eshed, Y., and Lifschitz, E. 1996. The making of a compound leaf: genetic manipulation of leaf architecture in tomato. Cell. 84(5): 735744. doi:10.1016/S0092-8674(00)81051-X. PMID:8625411.

Hay, A., Pieper, B., Cooke, E., Mandákova, T., Cartolano, M., Tattersall, A., loio, R., McGowan, S., Barkoulas, M., Galinha, C., Rast, M., Hofhuis, H., Then, C., Plieske, J., Ganal, M., Mott, R., Martinez-Garcia, J., Carine, M., Scotland, R., Gan, X., Filatov, D., Lysak, M., and Tsiantis, M. 2014. Cardamine hirsuta: a versatile genetic system for comparative studies. Plant J. 78(1): 1-15. doi:10.1111/tpj.12447. PMID:24460550.

Heuret, D., Barthélémy, D., Guédon, Y., Coulmier, X., and Tancre, J. 2002. Synchronization of growth, branching, and flowering processes in the South American 
tropical tree Cecropia obtusa (Cecropiaceae). Am. J. Bot. 89(7): 1180-1187. doi:10.3732/ajb.89.7.1180. PMID:21665718.

Jeune, B., and Lacroix, C. 2009. A quantitative analysis of leaf growth parameters in Myriophyllum aquaticum (Haloragaceae). Botany. 87(9): 807-820. doi:10.1139/B09-043.

Jones, A.W., Doughan, B.G., Gerrath, J.M., and Kang, J. 2013. Development of leaf shape in two North American native species of Ampelopsis (Vitaceae). Botany. 91(12): 857-865. doi:10.1139/cjb-2013-0210.

Kim, M., Pham, T., Hamidi, A., McCormick, S., Kuzoff, R., Sinha, N. 2003. Reduced leaf complexity in tomato wiry mutants suggests a role for PHAN and KNOX genes in generating compound leaves. Development. 130(18): 4405-4415.

doi:10.1242/dev.00655. PMID:12900456.

Letort, V., Heuret, P., Zalamea, P.-C., Nicolini, E., and de Reffye, P. 2009. Analysis of Cecropia sciadophylla morphogenesis based on a sink-source dynamic model. Third International Symposium on Plant Growth Modeling, Simulation, Visualization and Applications. doi:10.1109/PMA.2009.62.

Rast-Somssich, M.I., Broholm, S., Jenkins, H., Canales, C., Vlad, D., Kwantes, M., Bilsborough, G., Dello loio, R., Ewing, R.M., Laufs, P., Huijser, P., Ohno, C., Heisler, M.G., Hay, A., and Tsiantis, M. 2015. Alternate wiring of a KNOXI genetic network 
underlies differences in leaf development of $A$. thaliana and C. hirsute. Genes \& Development. 29(22): 2391-2402. doi:10.1101/gad.269050.115. PMID:26588991.

Reinhardt, D., Pesce, E., Stieger, P., Mandel, T., Baltensperger, K., Bennett, M., Traas, J., Friml, J., and Kuhlemeier, C. 2003. Regulation of phyllotaxis by polar auxin transport. Nature. 426(6964): 255-260. doi:10.1038/nature02081. PMID:14628043.

Rutishauser, R. 1998. Plastochrone ratio and leaf arc as parameters of a quantitative phyllotaxis analysis in vascular plants. In: Symmetry in Plants. Jean, R. V. and Barabé, D. (editors). World Scientific, Singapore, 836 pages.

Savolainen, V., Fay, M.F., Albach, D.C., Backlund, A., van der Bank, M., Cameron, K.M., Johnson, S.A., Lledó, M.D., Pintaud, J.-C., Powell, M., Sheahan, M.C., Soltis, D.E., Weston, P., Whitten, W.M., Wurdack, K.J., and Chase, M.W. 2000. Phylogeny of the eudicots: a nearly complete familial analysis based on rbcL gene sequences. Kew Bulletin. 55(2): 257-309.

Shani, E., Yanai, O., and Ori, N. 2006. The role of hormones in shoot apical meristem function. Curr. Opin. Plant Biol. 9(5): 484-489. doi:10.1016/j.pbi.2006.07.008. PMID:16877025.

Sicard, A., Thamm, A., Marona, C., Lee, Y.W., Wahl, V., Stinchcombe, J.R., Wright, S.I., Kappel, C., and Lenhard, M. 2014. Repeated evolutionary changes of leaf 
morphology caused by mutations to a homeobox gene. Curr. Biol. 24(16): 1880-1886. doi: 10.1016/j.cub.2014.06.061. PMID:25127212.

Sinha, H. 1999. Leaf development in angiosperms. Annu. Rev. Plant Phys. 50(1): 419446. doi:10.1146/annurev.arplant.50.1.419. PMID:15012215.

Sytsma, K., Morawetz, J., Pires, J., Nepokroeff, M., Conti, E., Zjhra, M., Hall, J., and Chase, M. 2002. Urticalean rosids: circumscription, rosid ancestry, and phylogenetics based on rbcL, trnL-F, and ndhF sequence. Am. J. Bot. 89(9): 1531-1546. doi:10.3732/ajb.89.9.1531. PMID:21665755.

Treiber, E.L., Gaglioti, A.L., Romaniuc-Neto, S., Madriñán, S., and Weiblen, G.D. 2016. Phylogeny of the Cecropieae (Urticaceae) and the evolution of an ant-plant mutualism. Syst. Bot. 41: 56-66. doi:10.1600/036364416X690633.

Uchida, N., Kimura, S., Koenig, D., and Sinha, N. 2010. Coordination of leaf development via regulation of KNOX1 genes. J. Plant Res. 123(1): 7-14. doi:10.1007/s10265-009-0248-2. PMID:19506991.

Vlad, D., Kierzkowski, D., Rast, M.I., Vuolo, F., Dello loio, R., Galinha, C., Gan, X., Hajheidari, M., Hay, A., Smith, R.S., Huijser, P., Donovan Bailey, C., and Tsiantis, M. 2014. Leaf shape evolution through duplication, regulatory diversification, and loss of a homeobox gene. Science. 343: 780-783. doi:10.1126/science.1248384. 
Wu, Z.-Y., Monro, A., Milne, R., Wang, H., Yi, T.-S., Liu, J., and Li, D.-Z. 2013. Molecular phylogeny of the nettle family (Urticaceae) inferred from multiple loci of three genomes and extensive generic sampling. Mol. Phylogenet. Evol. 69(3): 814-827. doi:10.1016/j.ympev.2013.06.022. PMID:23850510. 


\section{Figure legends}

Fig. 1. Mature leaves of Cecropia obtusa and Cecropia sciadophylla. (A) Cecropia obtusa, displaying a palmately-lobed leaf. (B) Cecropia sciadophylla, displaying a palmately-compound leaf. Scale bars $=30 \mathrm{~cm}$ (panel A), and $60 \mathrm{~cm}$ (panel B). Photographs courtesy of Denis Barabé, Institut de recherche en biologie végétale, Montréal, QC.

Fig. 2. Stages of leaf primordium morphogenesis in Cecropia obtusa (palmately-lobed) and Cecropia sciadophylla (palmately-compound) from initiation through to the end of the morphogenetic phase. Cecropia obtusa is shown in A, C, E, G, and I, and Cecropia sciadophylla in B, D, F, H, and J. A, B. Leaf initiation (P1) at the edge of the shoot apical meristem (SAM, asterisk). C, D. P1 growth flanking the SAM following initiation, associated stipule removed. E. Initiation of the first three lobes (arrowheads), on P2. F. Initiation of the first three leaflets (arrows), P2 stage. Note formation of stipules (S) at the base of the leaf primordium. G. Further development of lobes, showing the beginning of margin curling along edge of lobes, P3. H. Later stage of morphogenesis showing the initiation of additional leaflets, P3. I, J. End of the morphogenetic phase, showing curled margins and a full circular complement of lobes/leaflets on the adaxial surface, P4. Scale bars $=100 \mu \mathrm{m}($ panels $A-F), 150 \mu \mathrm{m}($ panels $\mathrm{G}$ and $\mathrm{H})$, and $500 \mu \mathrm{m}$ (panels I and J). 
Fig. 3. Dissection sequences of one specimen of each Cecropia obtusa (palmatelylobed) and Cecropia sciadophylla (palmately-compound), displaying leaf primordia and similarities in structure and organization at the shoot apex. C. obtusa is shown in A, C, E, and G, and C. sciadophylla in B, D, F, and H. A, B. P4 showing lobes (arrowheads), leaflets (arrows), and associated stipule (S), lateral view. Not all lobes/leaflets are labeled in order to reduce shadowing of adjacent tissue. C, D. P4 with associated stipule, top view. E, F. P4 and P3 stipules removed, revealing P2 at the three lobe stage and its associated stipule, P1, and the shoot apical meristem (SAM, asterisk). E. P1 is observed as a small raised bump opposite P2, adjacent to the SAM (asterisk), which is partially shielded by the developing P2 stipule. F. The SAM is visible as the smooth, flat central surface of the apex (asterisk), where the adjacent P1 is difficult to observe at the magnification and angle used. G, H. Fully dissected apices showing P1-P4, highlighting the high degree of similarity in SAM organization and scale between the two species, side view. Scale bars $=1 \mathrm{~mm}$.

Fig. 4. Measurement of phyllotactic parameters for Cecropia obtusa $(n=9)$ and Cecropia sciadophylla $(n=11)$ between successive leaf primordia at the shoot apex. A. Mean divergence angles. Asterisk, significant difference (two-sample t-test, $p=0.03$ ). B. Mean plastochron ratios. P1, youngest leaf primordium; P4, oldest leaf primordium. Error bars represent standard deviations. 


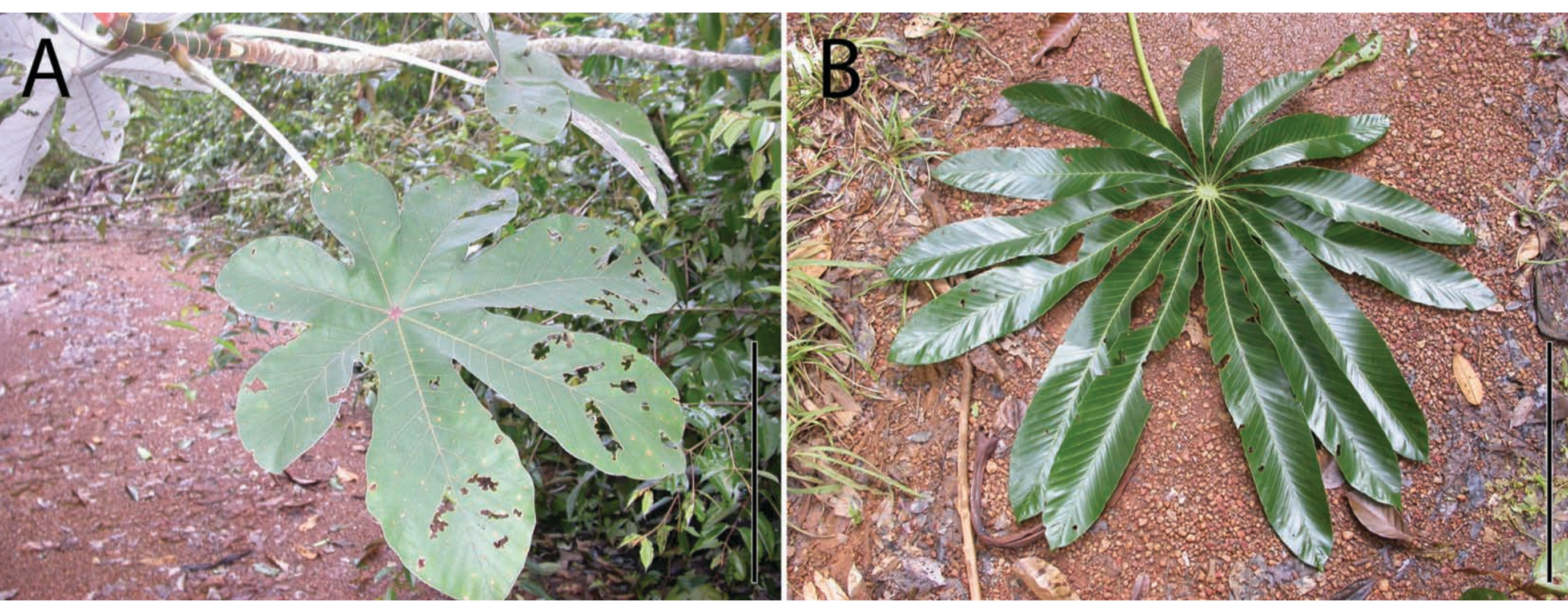



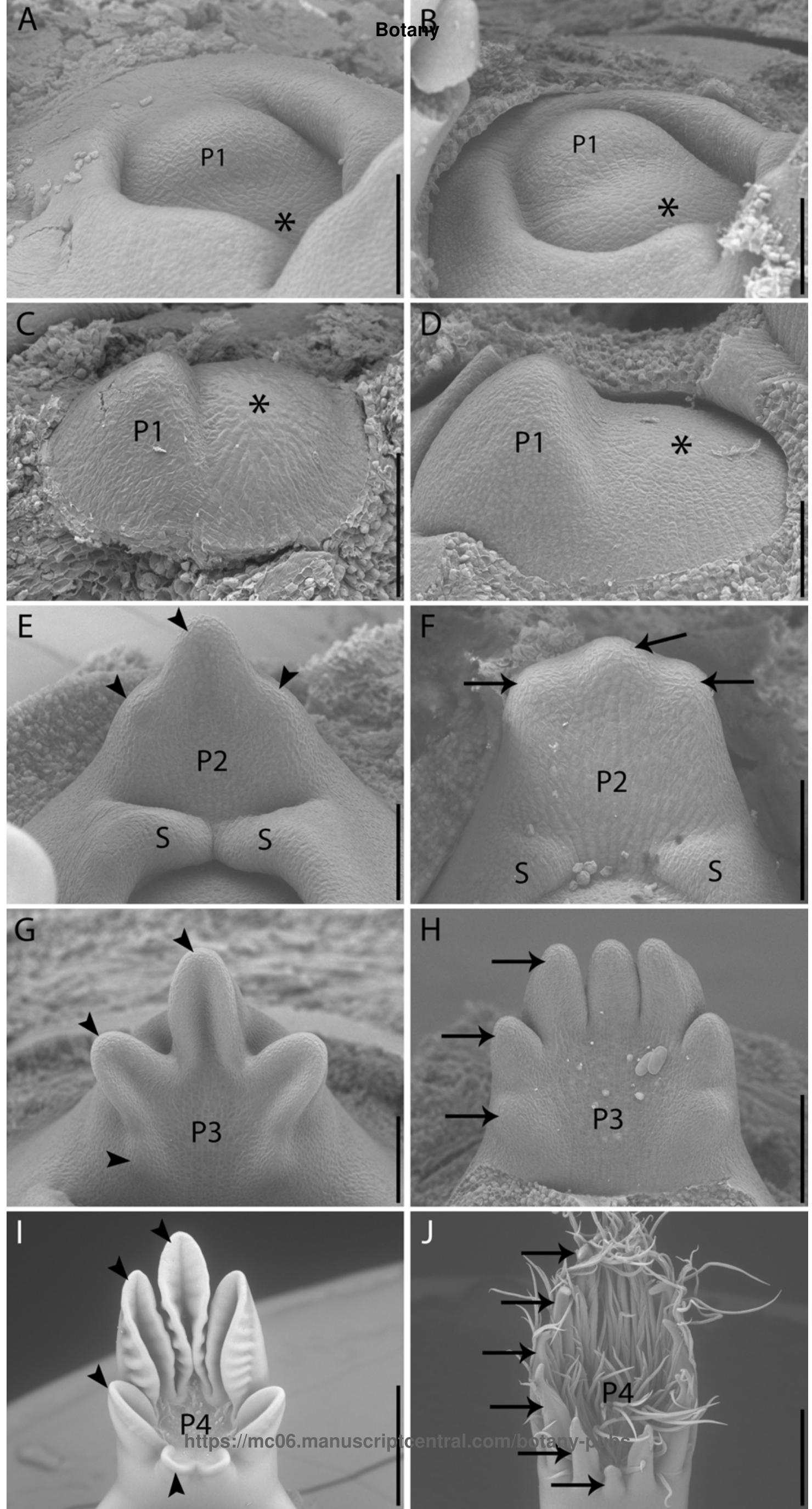

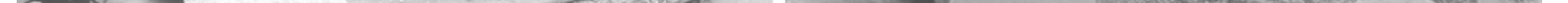

P4 
A

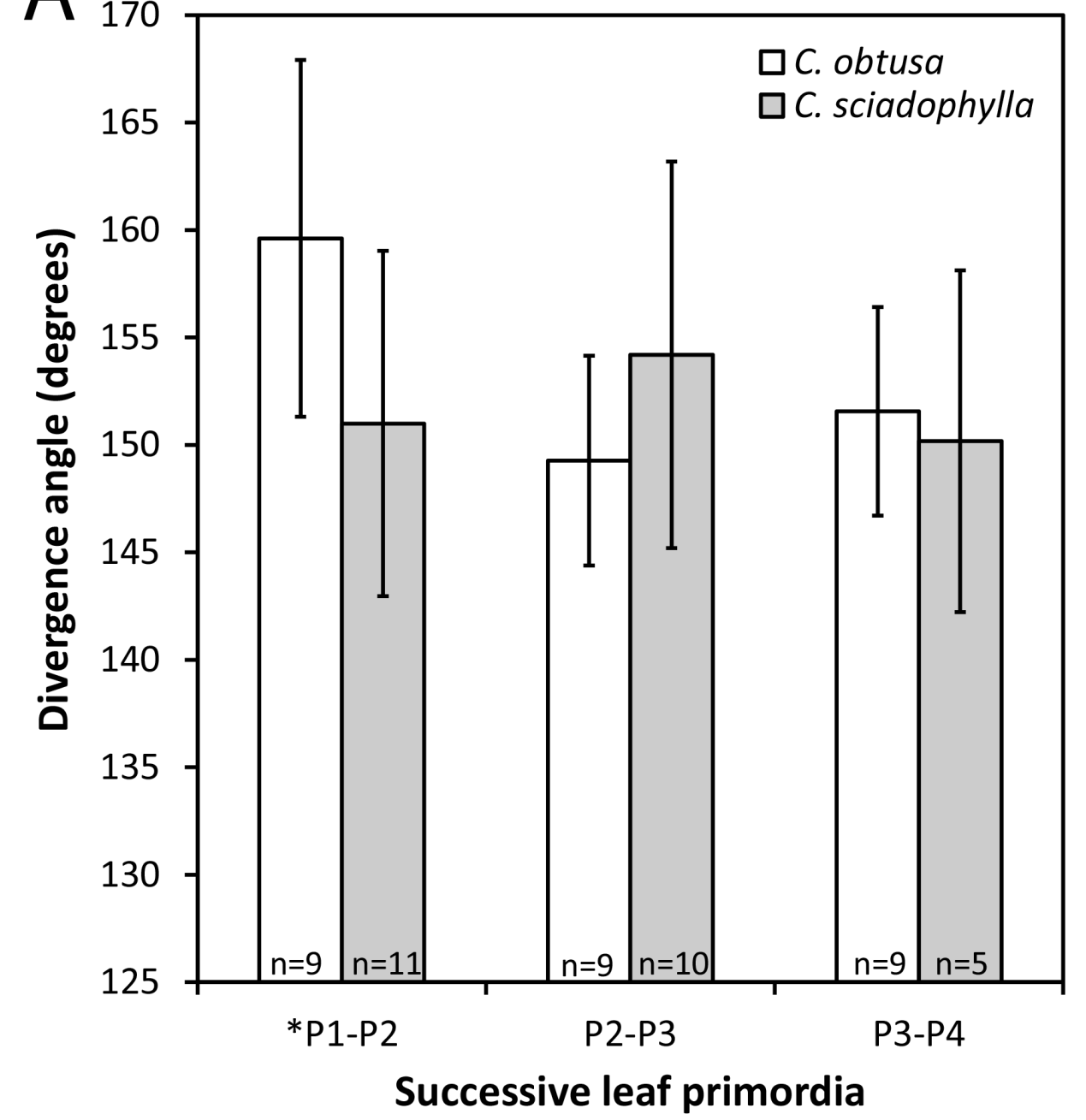

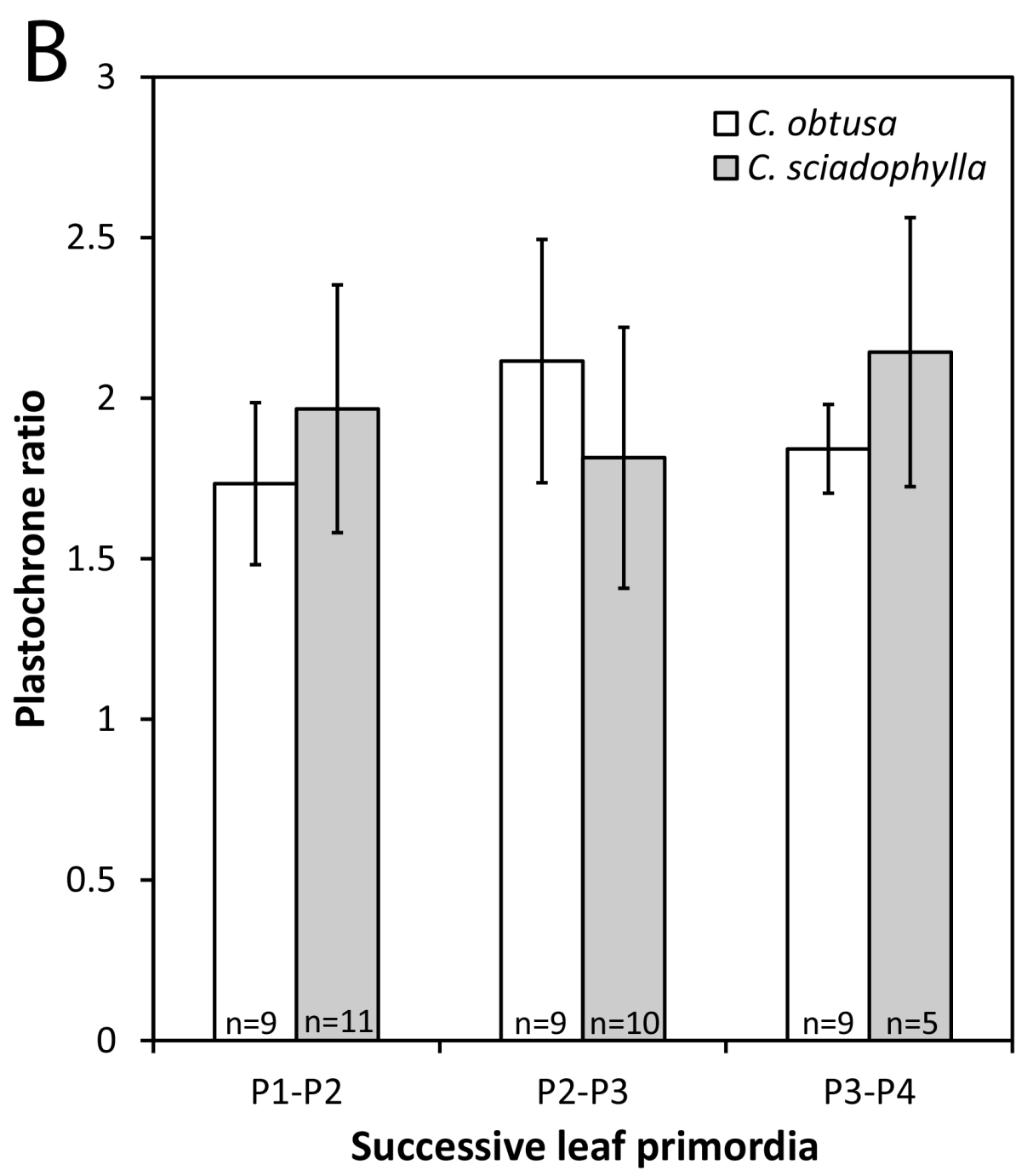

